\title{
O ENFRENTAMENTO DA COVID-19 NA ATENÇÃO PRIMÁRIA EM SAÚDE: UMA EXPERIÊNCIA EM NATAL-RN
}

\author{
Sheila Saint-Clair da S. Teodosio' \\ ORCID: 0000-0002-3169-029X \\ Maria Jacqueline Abrantes Gadelha" \\ ORCID: 0000-0001-7968-9952 \\ Meine Siomara Alcântara"II \\ ORCID: 0000-0003-4977-3189 \\ Márcia Lelis Rocha Correiaiv \\ ORCID: 0000-0002-5082-0002 \\ Maria de Lourdes Frederico Oliveira Freitas $\mathrm{v}$ \\ ORCID: 0000-0003-4816-2028 \\ Raul Brener Dantas ${ }^{\mathbf{v}}$ \\ ORCID: 0000-0002-6562-9737
}

' Universidade Federal do Rio Grande do Norte. Natal, Rio Grande do Norte, Brasil.

Coordenadora Nacional do Departamento Científico de Enfermagem na Atenção Básica-ABEn.

$\|\|, v,$,$v Secretaria Municipal de Natal.$ Natal, Rio Grande do Norte, Brasil.

v' Universidade Federal do Rio Grande do Norte. Natal, Rio Grande do Norte, Brasil. Discente, Bolsista de Iniciação Científica PIBC/UFRN.

Autor Correspondente: Sheila Saint-Clair da S. Teodosio Email: saintclairenf@gmail.com

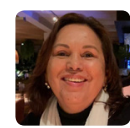

Como citar:

Teodosio SSCS, Gadelha MJA, Alcântara MS, Correia MLR, Freitas MLFO, Dantas RB. O enfrentamento da covid-19 na atenção primária em saúde: uma experiência em Natal-RN. In: Teodósio SSS,

Leandro SS (Orgs.). Enfermagem na atenção básica no contexto da COVID-19. 2.ed.rev. Brasília, DF : Editora ABEn, 2020. p. 49-54. (Série Enfermagem e Pandemias, 3). https://doi.org/10.51234/aben.20.e03.c07

\section{INTRODUÇÃO}

O novo coronavírus, denominado SARS-CoV-2, foi detectado em 31 de dezembro de 2019, no município de Wuhan, na China. Em nove de janeiro de 2020, a Organização Mundial da Saúde (OMS) confirmou a circulação do novo coronavírus e declarou, em 30 de janeiro de 2020, que o surto dessa doença constitui uma Emergência de Saúde Pública de Importância Internacional, conforme previsto no Regulamento Sanitário Internacional ${ }^{(1)}$.

Pela rapidez de sua disseminação em proporções globais, a Organização Mundial de Saúde (OMS), em 11 de março de 2020, a caracterizou como uma pandemia. Foram confirmados no mundo 11.500.302 casos de COVID-19 (172.512 novos em relação ao dia anterior) e 535.759 mortes (3.419 novas em relação ao dia anterior) até 7 de julho de $2020^{(1)}$.

No Brasil, o primeiro caso confirmado aconteceu no dia 26 de fevereiro de 2020, na cidade de São Paulo. Diante do avanço da COVID-19 no Brasil, o Ministério da Saúde (MS) criou o Grupo de Trabalho Interministerial de Emergência em Saúde Pública de Importância Nacional e Internacional para acompanhamento da situação e definição de protocolos de ação, para a vigilância do SARS-CoV-2 ${ }^{(2)}$.

Em 20 de março de 2020 foi confirmada a transmissão comunitária no Brasil e adotadas medidas de isolamento para casos leves e contatos, e de atenção hospitalar para os casos mais graves. As condições de vida precárias, principalmente nas periferias dos grandes centros urbanos, o agravamento das questões de saúde mental em decorrência do isolamento e a coexistência com outras morbidades, como as arboviroses, AIDS, tuberculose e doenças crônicas, ampliaram intensamente os desafios, exigindo mudanças de comportamento, atitudes colaborativas da sociedade e fortalecimento do sistema de saúde ${ }^{(3)}$.

Ademais, a pandemia chegou evidenciando as precárias condições de vida de grande parte dos brasileiros: são 35 milhões sem serviço de abastecimento de água 
e 100 milhões sem acesso a esgoto ${ }^{(4)}$. Estima-se que 122.890 pessoas estejam em situação de rua e, com o isolamento social, em decorrência do contingente de trabalho informal, cerca de 38 milhões de brasileiros ficaram sem renda ${ }^{(5)}$.

Nesse contexto, a pandemia instaurou a maior crise sanitária e humanitária do século, gerando uma sobrecarga no sistema de saúde de todos os países, como também no Brasil. Não obstante o Sistema Único de Saúde (SUS) ser um dos maiores sistemas de saúde universal do mundo, com uma extensa rede de Atenção Primária à Saúde (APS), seus problemas crônicos de financiamento, gestão, provisão de profissionais e estruturação dos serviço foram agudizados neste momento de crise que, para além da questão sanitária, tem uma relação com os campos político, social e econômico(6).

A APS é reconhecida como a base de sustentação do SUS, por ser a porta de entrada do sistema nacional de saúde e o primeiro elemento de um processo contínuo de atenção que visa atender às necessidades individuais/ familiares e coletivas em termos de promoção e proteção da saúde, prevenção de doenças, diagnóstico. Seu propósito está voltado para os cuidados essenciais à saúde, baseados em tecnologias que levam os serviços de saúde o mais próximo possível dos lugares de vida e trabalho das pessoas ${ }^{(7)}$. O modelo da APS brasileira, com suas equipes de saúde da família, territorialização e enfoque comunitário, tem um papel fundamental na rede de cuidados e pode contribuir no enfrentamento de qualquer epidemia ${ }^{(8)}$, inclusive no enfrentamento da COVID-19.

Diante do exposto, este estudo é norteado pelas seguintes questões: Como compatibilizar as ações pertinentes e o fio condutor do cotidiano da APS com a demanda proveniente da COVID-19? Como fazer a APS manter o fio condutor de suas atividades e (re)organizar um fluxo de atendimento para os sintomáticos respiratórios? Desse modo, o objetivo é relatar os desafios e os (re)arranjos das ações de enfermagem, na Atenção Básica, frente à Coronavirus Disease 2019, da Unidade Básica de Ponta Negra, no Município de Natal.

\section{TRAJETÓRIA METODOLÓGICA: MÉTODO E CENÁRIO DO ESTUDO}

Trata-se de estudo de abordagem qualitativa, descritiva, tipo relato de experiência, baseado em ações realizadas em combate à pandemia da COVID-19. Ele tem como cenário a Unidade de Saúde de Ponta Negra, no Município de Natal, capital do Rio Grande do Norte, e utilizou como referencial teórico os documentos e textos produzidos pela OMS, OPAS e MS.

\section{Cenário do estudo}

A Unidade de Saúde de Ponta Negra localiza-se no Município de Natal, capital do Estado do Rio Grande do Norte. É constituída por quatro equipes de Saúde da Família destinadas a oferecer cobertura de atenção à saúde para os moradores da Vila de Ponta Negra.

Assim como as demais USFs, a unidade de Ponta Negra tem como eixo condutor das suas ações a Estratégia Saúde da Família. Ela desenvolve cotidianamente ações de: pré-natal; acompanhamento de crescimento e desenvolvimento de crianças (CD); preventivos; visitas domiciliares às puérperas e acamados; consultas de acompanhamento às pessoas com tuberculose, doenças crônicas não transmissíveis e idosos; ações de imunização; saúde reprodutiva; grupo de gestantes; curativos; preceptoria dos alunos dos cursos da área de saúde públicas e privadas; acompanhamento do cadastramento dos Agentes Comunitários de Saúde no território; grupos de educação em saúde e saúde mental; entre tantas outras ações.

A unidade desenvolve ainda diversos projetos reconhecidos pela comunidade como importantes iniciativas de promoção e prevenção à saúde. Entre eles, destacam-se: Saúde na Praia (oferta de serviços e ações de educação em saúde junto aos trabalhadores da orla de Ponta Negra), Seminários sobre Envelhecimento Saudável, Revita Vila (revitalização de espaços públicos em situação de abandono), PICS na Vila (Práticas Integrativas e Complementares junto à comunidade), Encontros e Afetos (grupo de mulheres com foco na saúde mental). 
Os resultados descritos a seguir tiveram como base as experiências da USF, em andamento, relatadas pelas enfermeiras autoras do estudo.

\section{RESULTADOS DA EXPERIÊNCIA E DISCUSSÃO}

\section{Os desafios e (re)arranjos das ações da Unidade de Saúde}

Com a instalação da pandemia, no Município de Natal, em março de 2020, os profissionais da APS foram surpreendidos por novas demandas e novos protocolos, principalmente a partir da transmissão comunitária. O grande desafio foi compatibilizar as ações pertinentes ao cotidiano da APS com a demanda proveniente da COVID-19.

Por se entender que APS/ESF é a porta de entrada do Sistema Único de Saúde, durante surtos e epidemias, e tem papel fundamental na resposta à doença em questão(1), era premente a necessidade de se (re)inventar processos de trabalho, estabelecer novos fluxos e fortalecer redes de apoio.

Acompanhavam-se com temor nos noticiários da TV a proximidade da morte, os riscos e o perigo das relações sociais expressadas pela inexistência de visitas nos hospitais, o não direito a despedidas, velórios limitados, enfim, um luto com mais de uma lacuna na dor. Impossibilitados de reunir pessoas nos grupos, de se aproximar, de tocar as mãos, de acolher com um sorriso ou com um abraço, os profissionais foram sendo tomados pelo medo, pelas incertezas e pela ausência de respostas às mais diversas inquietações: Como enfrentar o medo? Como cuidar? Como adequar as rotinas às novas demandas?

\section{Mudanças no Processo de Trabalho: desafios da equipe em saúde}

Logo que os primeiros casos chegaram ao Brasil e, em especial, ao Rio Grande do Norte, foi realizada uma reunião multidisciplinar para definição do novo fluxo de atendimento, considerando-se que a APS/ESF deve oferecer atendimento resolutivo, além de manter a longitudinalidade e a coordenação do cuidado em todos os níveis de atenção à saúde, e assim conseguir grande potencial de identificação precoce de casos graves que devem ser manejados em serviços especializados, conforme protocolos do $\mathrm{MS}^{(9)}$.

Foram formadas equipes de acolhimento que ficaram responsáveis pela recepção dos usuários, detectar os sintomáticos e encaminhá-los para atendimento por uma equipe constituída por um médico, um técnico de enfermagem e um agente comunitário de saúde, seguindo as regras de isolamento e uso de equipamentos de proteção individuais (EPIs). Dessa forma, para evitar aglomeração e proteger as pessoas de maior grau de risco, nesse primeiro momento, foram suspensas algumas ações realizadas na USF, dentre as quais, a coleta de citologia oncótica, saúde bucal, acompanhamento à criança sadia (Crescimento e Desenvolvimento), testes rápidos para HIV, Sífilis, Hepatite B e Hepatite $C$, exceto para as gestantes ou alguma urgência.

O acompanhamento das pessoas atendidas com suspeitas por COVID-19 passou a ser realizado através de telefonemas diários, com os dados colocados em uma planilha elaborada por um profissional da USF, além dos casos notificados no e-SUS VE. A ESF incluiu medidas de suporte e conforto para casos leves, isolamento domiciliar e monitoramento até a alta do isolamento. Para casos graves, incluiu a estabilização clínica e o encaminhamento e transporte a centros de referência ou serviços de urgência/emergência ou hospitalares ${ }^{(9)}$.

Concomitantemente com a chegada da pandemia do COVID-19, iniciou-se a vacinação da influenza que, este ano, foi antecipada para o mês de março, começando pelos idosos e profissionais de saúde. Naquele momento, foi preciso retirar as pessoas idosas da Unidade e solicitar a parceria com as organizações sociais da comunidade para cederem seus espaços como locais da administração da vacina. Dentre os locais destaca-se o Centro Social Estevam Pascoal e a Escola Estadual Jerônimo de Albuquerque na Vila, e a Associação dos Moradores de Ponta Negra (AMPA), localizada no conjunto Ponta Negra. Logo nos primeiros dias aumentou consideravelmente a demanda, causando preocupação, e por questões de segurança fez-se necessário adotar 
medidas de) distanciamento e de higiene nos locais escolhidos. Logo em seguida iniciou-se a segunda etapa da vacina para outros grupos e foi possível conseguir alcançar o objetivo proposto de imunizar a população da área de abrangência.

\section{(Re)Arranjos e (Re)Invenções: ações da equipe em tempos da COVID-19}

\section{Fortalecimento de redes de apoio e parcerias relevantes}

Conforme dados do E-SUS 2014(9), os determinantes sociais preponderantes da Vila de Ponta Negra são: o desemprego e o subemprego, trabalhadores autônomos sem carteira assinada, moradias insalubres, além de predominarem como principais situações de saúde a obesidade, o tabagismo e a hipertensão, ou seja, moradores em situação de grande vulnerabilidade social. Diante desses dados, dá para se imaginar a dificuldade que a maioria dos moradores tem para cumprir as medidas de higienização, uso de máscaras e de isolamento.

Nesse contexto, as parcerias institucionais e a articulação de redes de apoio comunitárias e ações de solidariedade foram, sobremaneira, essenciais para o desenvolvimento de atividades que compuseram os (re)arranjos neste momento de pandemia. Destaca-se o fundamental apoio institucional da Universidade Federal do Rio Grande do Norte (UFRN), através da produção e distribuição de EPIs, materiais instrucionais diversos (protocolos, notas técnicas, manuais e cartilhas), organização de lives educativas e criação do teleatendimento aos idosos do território da UBS.

Com o avanço dos casos da Covid-19 e as recomendações do MS, fez-se necessário adotar medidas de proteção à saúde dos profissionais e usuários e apoiar os últimos para adotarem as medidas de higiene. Sabendo-se das condições precárias da maioria dos usuários da unidade, iniciou-se uma campanha para doação de máscaras e de material para montar kits higiênicos (água sanitária, sabão em barra, sabonete e máscaras de tecido). $O$ apoio de amigos, professores de universidade, comércio local e dos funcionários permitiu a entrega de mais de 400 kits, havendo a possibilidade de mais pessoas serem beneficiadas, já que a campanha ainda não foi concluída.

\section{Projeto Cuca Legal}

Este projeto surgiu da preocupação da equipe com relação ao isolamento social das pessoas idosas, no confinamento havia mais de três meses, pelo grande número de informações negativas de familiares sobre mudança de comportamento do idoso. Consiste em promover atividades de estimulação cognitiva, o resgate da criatividade através de atividades de pintura, e incentivar a realização de exercícios físicos. Os idosos recebem, em suas casas, uma cartilha com orientações de exercícios físicos elaborada pelos alunos e professores da UFRN junto com um kit (pasta, lápis grafite, borracha, apontador, lápis de cor). Semanalmente levam-se novos exercícios e se averigua como estão sendo feitos e a receptividade do idoso, aproveitando-se para saber sobre a situação de saúde. A partir dessas visitas, já se tem alguns resultados preliminares relatados pelos familiares, que afirmam o quão os idosos estão satisfeitos. Ademais, este projeto tem recebido muitos elogios da imprensa local.

\section{Vigilância à pessoa idosa}

A USF também participa da vigilância aos idosos, através de busca ativa realizada por estudantes de diversas universidades públicas e privadas. Diariamente os estudantes ligam para as pessoas idosas, e procuram saber como elas se encontram, quais seus principais anseios, se têm alguma necessidade específica, seja de saúde ou social, entre outras. Teve início em maio de 2020, sendo um subprojeto do projeto do Teleatendimento à COVID-19, uma parceria da UFRN e Secretaria Municipal de Saúde de Natal, Secretaria do Estado da Saúde Pública (SESAP). Uma enfermeira e dois agentes comunitários de saúde da USF Ponta Negra fazem a ponte 
entre as necessidades colhidas pelos alunos e a USF, procurando dar respostas com maior brevidade. Dentre as várias demandas, solicitadas e percebidas pelos alunos, encontraram-se idosos em vulnerabilidade social, nutricional, por violência doméstica, necessidades de consultas médicas e solidão.

\section{A Vila em movimento: a sala de situação}

O Fórum Vila em Movimento consiste em uma rede cidadã, de articulação e mobilização social de indivíduos, organizações e instituições, cujo objetivo é lutar pela garantia e defesa dos direitos humanos e socioambientais. A partir das reuniões do Grupo de Trabalho desse Fórum, surgiu a ideia de uma rede de comunicação para atuar na área da Vila de Ponta Negra. Desse modo, surgiu a Sala de Situação, que tem como objetivo realizar ações preventivas e orientações, além de monitorar dados oriundos do acompanhamento dos sintomáticos respiratórios atendidos na Sala de Priorização de Atendimento (SPA) da USF. Dentre as diversos criações desta sala, destacam-se vídeos educativos e folders produzidos por moradores, artistas e profissionais de saúde vinculados às diversas redes sociais; a construção de mapas para saber em quais ruas acontecem os maiores números de casos; criação do grupo de WhatsApp Comunica Vila, além de colocação de faixas com frases sobre a COVID-19, em todos os recantos da Vila e adjacências. Esse grupo se reúne semanalmente para avaliação das ações e planejamento de novas estratégias de enfrentamento à Covid-19.

\section{Comunica Vila}

A partir da decretação do distanciamento social, em virtude da pandemia, a equipe de saúde sentiu a necessidade de levar informações às pessoas da Vila, incluindo as mais vulneráveis. Assim, foi criado o grupo de WhatsApp Comunica Vila. Ele é um grupo fechado, no qual diariamente coloca-se, pela manhã, uma mensagem de cunho educativo e, no final da tarde, uma mensagem afetiva, de valorização da pessoa idosa, de esperança, e músicas que tocam o coração. O grupo foi formado com os contatos das pessoas idosas e familiares fornecidos pelos agentes comunitários de saúde.

Vale ressaltar que esses projetos já estão recebendo o reconhecimento dos usuários e familiares, bem como da imprensa local. Tal fato, demonstra a importância e a validade das ações desenvolvidas, além de potencializar a contribuição da APS/ESF em vista de suas iniciativas para o enfrentamento da epidemia.

No entanto, convém destacar que, embora o Brasil tenha um Sistema Único de Saúde (SUS) reconhecido mundialmente, pela funcionalidade da sua rede de APS, um dos maiores desafios enfrentados pela equipe da USF, objeto deste estudo, diz respeito à falta de equipamentos e condições adequadas para o desempenho de suas atividades em decorrência da falta de financiamento adequado para a APS.

A literatura mostra que a crise atual da saúde não se restringe a uma questão sanitária, ela está relacionada com os campos político, social e econômico. "A força da APS não é apenas um espaço de triagem e de acompanhamento de casos leves da COVID-19, as USFs precisam receber investimentos para cumprir adequadamente o seu papel"(10). Urge a centralização da APS na agenda do MS e que o SUS não seja asfixiado com cortes no seu financiamento. "O sucesso do enfrentamento à Covid-19, o futuro do SUS e a saúde dos brasileiros também dependem disso"(6). Destaca-se ainda a necessidade da luta pelo fortalecimento do SUS e a revogação imediata da Emenda Constitucional 95, que ao não aprovar o financiamento universal do SUS, desconsiderou um dos seus princípios básicos; a universalidade. Tal fato compromete, sobremaneira, a garantia dos serviços de saúde a população em geral, e em particular àqueles que dependem unicamente do SUS.

\section{CONSIDERAÇÕES FINAIS}

O presente estudo teve o propósito de evidenciar as experiências da equipe de saúde de uma USF no contexto da COVID-1. O seu desenvolvimento permitiu compreender que, mesmo na adversidade, a equipe 
de saúde (re)inventou ações para garantir a atenção à saúde, tanto aos usuários da demanda pertinente aos protocolos da Estratégia da Saúde da Família, quanto àqueles com suspeita de COVID-19.

Vale desatacar que as enfermeiras da USF estão na linha de frente, demonstrando seu compromisso no enfrentamento da COVID-19, como parte da equipe de saúde, além de dar continuidade às ações cotidianas para garantir a atenção aos indivíduos e famílias, grupos e comunidades das suas áreas adstritas, dentro do território.

Embora este trabalho esteja centralizado em relatos de experiências de uma única USF, espera-se que ele possa incentivar outras publicações mais abrangentes.

Portanto, almeja-se que este estudo possa estimular a socialização de experiências exitosas desenvolvidas pelas USFs/ESF, mesmo vivenciando situações adversas, e contribuir para o reconhecimento do protagonismo da APS como ordenadora do cuidado no SUS.

\section{REFERÊNCIAS}

1. Organização Pan-Americana de Saúde (OPAS). Folha informativa - COVID-19 (doença causada pelo novo coronavírus). Brasília (DF): OPAS; 2020 [cited 2020 Jun 28]. Disponível em: https://www.paho.org/bra/covid19/

2. Lana RM, Coelho FC, Gomes MFC, Cruz OG, Bastos LS, Villela DAM, et al. Emergência do novo coronavírus (SARS-CoV-2) e o papel de uma vigilância nacional em saúde oportuna e efetiva. Cad Saúde Pública [Internet]. 2020 [cited 2020 Jun 28]; 36(3): e00019620. Doi: 10.1590/0102-311x00019620

3. Oliveira WK, Duarte E, França GVA, Garcia LP. Como o Brasil pode deter a COVID-19. Epidemiol Serv Saúde [Internet]. 2020 [cited 2020 Jun 28]; 29(2): e2020044. Doi: 10.5123/s1679-49742020000200023

4. Instituto Trata Brasil. Saneamento é saúde. Principais estatísticas no Brasil; 2020 [cited 2020 Jun 29]. Disponível em: http:// www.tratabrasil.org.br/saneamento/principais-estatisticas/no Brasil/agua.

5. OXFAM Brasil. Pandemia de coronavírus reforça desigualdades da população mais vulnerável; 2020 [cited 2020 Jun 28]. Disponível em: https://www.oxfam.org.br/blog/pandemia-de-coronavirus-reforca-desigualdades-da-populacao-mais-vulneravel/

6. Sarti TD, Lazarini WS, Fontenelle LF, Almeida APSC. Qual o papel da Atenção Primária à Saúde diante da pandemia provocada pela COVID-19? Epidemiol Serv Saúde [Internet]. 2020 [cited 2020 Jun 28]; 29(2):e2020166. Doi: 10.5123/ s1679-49742020000200024

7. Organização Pan-Americana da Saúde (OPAS). Declaração de Alma-Ata; 2015 [cited 2020 Jun 22]. Disponível em: https:// opas.org.br/declaracao-de-alma-ata/

8. Brasil. Ministério da Saúde. Secretaria de Atenção Primária à Saúde. Protocolo de Manejo Clínico do Coronavírus (COVID-19) na Atenção Primária à Saúde - Versão 9. Brasília (DF): Ministério da Saúde; 2020 [cited 2020 Jun 22]. Disponível em: https://pesquisa.bvsalud.org/portal/ resource/pt/biblio-1087335

9. Giovanella L. APS na rede de enfrentamento da Covid - 19. Centro Brasileiro de Estudos da Saúde; 2020 [cited 2020 Jun 30]. Disponível em: http://cebes.org.br/2020/04/aps-na-rede-de-enfrentamento-da-covid-19/

10. Vitória AM, Campos GWS. Só com APS forte o sistema pode ser capaz de achatar a curva de crescimento da pandemia e garantir suficiência de leitos UTI. Conselho de Secretarias Municipais de Saúde de São Paulo; 2020 [cited 2020 Jun 27]. Disponível em: http://www.cosemssp.org.br/wp-content/uploads/2020/04/So-APS-forte-para-ter-leitos-UTI-.pdf 